\title{
DIGITALIZACIÓN, TIEMPO DE TRABAJO Y SALUD LABORAL
}

\author{
Mariola Serrano Argüeso ${ }^{1}$ \\ Profesora Titular de Derecho del Trabajo y de la Seguridad Social \\ Universidad de Deusto. Bilbao
}

\begin{abstract}
La tecnología es neutra, su utilización no. En la era del trabajo digital e inteligente los empleados son los dueños de su tiempo, pero el mal uso de las herramientas tecnológicas puede conllevar prolongaciones de la jornada de trabajo, interferencias o invasiones en la vida familiar y personal y, en consecuencia, daños en la salud de los trabajadores. En este artículo se analizan las medidas de prevención que el empresario debe adoptar para garantizar el descanso de los trabajadores y una conciliación corresponsable, a la luz del derecho a la desconexión digital.

Technology is neutral but its utilization is not. In the digital and intelligent age, employees are their own time owners but the technology tools bad usage can imply working hours extension, interferences or invasions in in the family and personal life and, consequently, damages in the workers' health. In this article, the prevention measures that an employer has to adopt in order to guarantee the workers rest and a co-responsible conciliation are analyzed based on the digital disconnection right.
\end{abstract}

Title: Digitalization, working time and occupational health.

Palabras clave: digitalización, tiempo de trabajo, prevención, salud laboral, conciliación corresponsable, desconexión digital.

Key words: Digitalization, working time, prevention, occupational health, co-responsible conciliation, digital disconnection.

IUSLabor 2/2019, ISSN 1699-2938, p. 8-31

DOI. 10.31009/IUSLabor.2019.i02.02

\footnotetext{
${ }^{1}$ El artículo está vinculado al Proyecto de Investigación: Trabajo y Digitalización: el impacto de la economía 4.0 sobre el empleo, las relaciones laborales y la protección social DER2017-82444-R.
} 


\section{Sumario}

1. Hiperconexión digital y salud laboral

2. El nuevo derecho a la desconexión digital

3. ¿Cómo garantizar el derecho al descanso de los trabajadores y una conciliación corresponsable?

4. Bibliografía 


\section{Hiperconexión digital y salud laboral}

Las tecnologías de la información y de la comunicación, en general, e internet, en particular, están modificando radicalmente las relaciones laborales. Esta tendencia parece que no va a hacer más que incrementarse en el futuro generando así cambios sustanciales en la organización de las tareas, del tiempo de trabajo, de las interrelaciones sociales o de la gestión empresarial ${ }^{2}$. No cabe duda de que las nuevas tecnologías facilitan el trabajo y favorecen la productividad y eficiencia, pero modifican también los parámetros tradicionales del tiempo de trabajo promoviendo la autogestión, así como la realización del trabajo en cualquier momento debido a la facilidad de localización y de conexión permanente. Presentan, además, un "potencial alienante" y riesgos laborales de primera magnitud no por la tecnología que es, en sí, neutra, sino por su uso que debe ser racional lo que, a su vez, requiere también de aprendizaje y experiencia a fin de responder al reto de garantizar un equilibrio entre la seguridad y salud de los trabajadores y la adecuada implantación de los nuevos cambios organización que demandan una mayor flexibilidad laboral, el uso intensivo de las TICs y la virtualización de las relaciones laborales ${ }^{3}$. A diferencia de lo que ocurre en Europa, dónde existe una legislación sobre seguridad y salud laboral de carácter muy avanzado que establece unos criterios mínimos cuya aplicación y mejora continua se deja en manos de la colaboración entre empresarios y trabajadores, en España, la aplicación de esta legislación se ha visto lastrada por un modelo de relaciones laborales caracterizado por una elevada conflictividad, un enraizado autoritarismo empresarial y unos sindicatos históricamente politizados y divididos. De esta forma, si la integración europea no se acompaña de una integración de los sistemas de relaciones laborales, se puede dar una pérdida de las conquistas sociales, en particular en cuanto a salud laboral ${ }^{4}$.

Los grandes ejecutivos de Google, Twitter y Facebook están apagando sus dispositivos móviles y desconectándolos de la red. No es una cuestión baladí. No hay una definición científica ni legal de hiperconexión digital ni tampoco de desconexión digital pero sí se advierten algunas manifestaciones adictivas del primero de los fenómenos como, por ejemplo, obsesión por perderse algo de lo que está sucediendo en la red, adicción al WhatsApp, ansiedad al teléfono móvil o utilización enfermiza del Smartphone. La tecnología, como se ha señalado, es imparcial, su uso no, y las consecuencias de una incorrecta utilización se extienden también al ámbito laboral. En este sentido, se ha señalado que una vez que el trabajo se lleva a la vida privada las personas se vuelven más irritables y tienden a ser más propensas a sufrir enfermedades y/o accidentes causados

\footnotetext{
${ }^{2}$ Centro Nacional de Condiciones De Trabajo, "El futuro de la prevención”, NTP 1000, INSHT, 2014.

${ }^{3}$ FERNÁNDEZ AviLÉs, J. A., "NTC y riesgos psicosociales en el trabajo: estado de situación y propuestas de mejora", $D S L, \mathrm{n}^{\circ} 2,2017$, p. 70-72,

${ }^{4}$ Centro Nacional de Condiciones De Trabajo, "El futuro de la prevención”, op. cit.
} 
por el deterioro en su calidad de vida ${ }^{5}$ Un informe del entonces INSHT $^{6}$ considera que estar permanentemente conectado provoca insomnio, irritabilidad, mal humor, desmotivación, agotamiento mental, falta de energía y menor rendimiento. Tristemente, el problema de la conexión permanente no es, sin embargo, excepcional en el ámbito empresarial. Según se desprende de los resultados de un estudio elaborado por la empresa ENDENRED y la consultoría IPSOS un $65 \%$ de los trabajadores reconoció que su empresa le llamaba fuera del horario laboral. Entre los directivos, la cifra se disparaba hasta el $90 \%$, No solo eso, el $98 \%$ de los trabajadores reconocieron enviar emails de noche o en fin de semana, un 53\% que se levantaba mientras comía por una llamada de trabajo y tan solo un $3 \%$ que no enviaba emails de trabajo en sus vacaciones ${ }^{7}$. Así pues, cabe reseñar que uno de los aspectos más inquietantes del uso intensivo de las TICs en el trabajo es que habilitan contextos laborales en los que a falta de sensibilización y formación para la gestión adecuada del tiempo resulta fácil caer en la "trampa" de lo que podría denominarse libertad para auto explotarse, pensando que se es libre ${ }^{8}$. También en este contexto nuevo y futuro, advierte el Centro Nacional de Condiciones de Trabajo que para abordar los problemas de salud laboral del siglo XXI hay que actuar sobre las patologías no incluidas en las enfermedades profesionales, asociadas a una población más feminizada y envejecida, al sector servicios y a las nuevas tecnologías de la comunicación, con un tamaño menor de las empresas y mayor de desregulación contractual y dónde difícilmente vamos a poder desligar la causa laboral de los estilos de vida y otros factores extra laborales ${ }^{9}$.

La cotidianidad en el uso de las TIC en el ámbito laboral expone la NTP 1122, está relacionada con el proceso de globalización del mercado laboral y el aumento de la competitividad, que han requerido a las empresas mayor flexibilidad en la organización del trabajo y ha dado lugar a nuevas formas de organización diferentes a las tradicionales. Éstas suponen un desafío para los técnicos de prevención, ya que incorporan cambios en la concepción tradicional del espacio físico dónde se realiza el trabajo y en los tiempos de trabajo (impulsando la autogestión) con el riesgo de prolongar la jornada laboral

\footnotetext{
${ }^{5}$ HERNÁNDEZ, J. A. et al., "Las tecnologías de la Información y Comunicación y la Brecha Digital: Una nueva forma de exclusión social", Boletín Científico de la Escuela Superior de Atotonilco de Tula, $\mathrm{n}^{\circ}$ 11, 2019, (última consulta: 15/05/ 2019), Disponible en: https//repository.uaeh.edu.mx/revistas/index.php/Atotonilco/issue/ archive

${ }^{6}$ Ahora Instituto Nacional de Seguridad y Salud en el Trabajo (INSST).

${ }^{7}$ EDENRED-IPSOS, Bienestar y motivación de los empleados, junio 2015, (última consulta: 4/6/2019), Disponible en: www.edenred.es/descarga-barometro-edenred-ipsos

${ }^{8}$ FERNÁNDEZ AVILÉs, J. A., "NTC y riesgos psicosociales en el trabajo: estado de situación y propuestas de mejora", op. cit., p. 70-72.

${ }^{8}$ FERNÁNDEZ AVILÉS, J. A., "NTC y riesgos psicosociales en el trabajo: estado de situación y propuestas de mejora", op.cit., p. 74.

${ }^{9}$ Centro Nacional de Condiciones De Trabajo, "El futuro de la prevención”, op.cit.
} 
debido a la facilidad para la conexión en cualquier momento y lugar, incidiendo así en la conciliación de la vida laboral, personal y familiar. En su manifestación más extrema puede suponer la supresión de los límites entre la vida personal y el trabajo (efecto blurring ${ }^{10}$. Ante un problema que empieza a visibilizarse con fuerza, el Nuevo Marco Estratégico Europeo de Seguridad y Salud en el Trabajo 2014-2020 insta a evaluar los cambios en la organización del trabajo derivados de la evolución de las nuevas TICs (conectividad constante) y su impacto a nivel organizacional e individual por los efectos que a medio plazo puede tener en la salud de los trabajadores. En efecto, cuando las condiciones de trabajo relacionadas con la organización, el contenido del trabajo y la realización de la tarea están configuradas deficientemente o con un diseño inadecuado podrían causar malestar y/o daño y, por tanto, se convierten en factores de riesgo psicosocial, que, si se dan los requisitos, pueden contribuir a la generación de un riesgo psicosocial propiamente dicho ${ }^{11}$.

La conectividad permanente que facilitan las nuevas tecnologías posibilita la confusión entre conectividad y disponibilidad lo que puede producir la prolongación de la jornada de trabajo más allá de lo legalmente permitido. El hecho de que las personas trabajen cada vez más fuera de los lugares de trabajo tradicionales o lo hagan a distancia aleja a los trabajadores de los lugares de trabajo peligrosos, pero también puede situarlos frente a nuevos riesgos. Los factores psicosociales y organizativos adquieren cada vez más importancia, al igual que lo hace el tipo y el ritmo de trabajo, incluido el modo en que (se gestionan los cambios ${ }^{12}$. La NTP $1122^{13}$ incorpora un listado no exhaustivo de factores de riesgo psicosocial por el uso inadecuado y no planificado de las TICs relacionados con el tiempo de trabajo:

- Prolongación de la jornada laboral (antes de su comienzo o al finalizar la misma) y realización del trabajo en horarios inusuales (noche, fin de semana etc.) debido a la conectividad permanente y al uso inadecuado de las TICs.

- Dificultad de conciliar la vida familiar y laboral.

- Aumento de las interrupciones en el desarrollo del trabajo, en especial en el uso del correo electrónico.

- Tareas no previstas y multitarea que pueden prolongar la jornada laboral.

\footnotetext{
${ }^{10}$ Manzano Santamaría, N., "Las Tecnologías de la Información y la Comunicación (TIC)(I): nuevas formas de organización del trabajo", NTP 1122, INSSBT, 2018.

${ }^{11}$ Instituto Nacional de Seguridad, Salud y Bienestar en el Trabajo (INSSBT), Algunas orientaciones para evaluar los factores de riesgo psicosocial, Torrelaguna (Madrid), 2015.

${ }^{12}$ Disponible en: https://osha.europa.eu

${ }^{13}$ FERNÁNDEZ AviLÉs, J. A., "NTC y riesgos psicosociales en el trabajo: estado de situación y propuestas de mejora", op. cit., p. 74.

${ }^{13}$ ManZano Santamaría, N, "Las Tecnologías de la Información y Comunicación (TIC) (II): factores de riesgo psicosocial asociados a las nuevas formas de organización del trabajo", op. cit.
} 
- Realización de tareas en "periodos de espera" o "tiempos muertos" (entre desplazamientos y/o viajes) que antes eran "inactivos" (ya que no se podía realizar el trabajo sin estar presente en la oficina) y ahora son trabajados y pueden suponer un añadido al tiempo de trabajo de la jornada laboral.

- Asincronismo temporal para interactuar con otros trabajadores ubicados en países con diferentes franjas horarias, lo que incide en la jornada laboral, prolongando o adelantando el comienzo de la misma.

- Cultura de empresa sin una política clara sobre promoción de trabajadores remotos, lo que hace aumentar la competitividad, pudiendo ocasionar un aumento de las horas de trabajo usando las TICs fuera del horario laboral y del centro de trabajo, ocasionando a su vez prácticas laborales inadecuadas como el leaveism (entendido en su acepción de realización del trabajo en el hogar, fuera de la jornada laboral, para adelantar trabajo sin dar parte de esta práctica ni a compañeros ni a superiores jerárquicos).

- Inestabilidad laboral y ausencia de cultura empresarial clara entorno al uso inadecuado de las TICs fuera de la jornada laboral como el leaveism (entendido en su acepción de llevar el trabajo "al día" y realizarlo fuera de la jornada laboral para lograr consolidar el puesto de trabajo ante la amenaza de una no renovación o despido).

- Cultura empresarial que no tenga en cuenta la movilidad física constante para la realización del trabajo (a nivel local, autonómico, nacional o internacional en países con o sin diferentes franjas horarias) como tiempo de trabajo y/o la prolongación de la jornada laboral que ello pueda suponer.

Es conveniente desechar mitos porque el uso de las tecnologías en el trabajo no necesariamente nos libra del trabajo monótono, repetitivo e insatisfactorio, esto es, no reemplaza la necesidad de un trabajo que dé sentido a nuestra vida profesional, como tampoco cubre la necesidad de una red de apoyo en el trabajo ni libera de la sensación de soledad y de aislamiento en el nuevo mundo de la hiperconexión y las redes sociales ${ }^{14}$. Amazon, por ejemplo, ha patentado una muñequera que localiza a los trabajadores de los almacenes y vibra para dirigirlos hacia el lugar de su nueva tarea. Muchos sienten que la principal interacción que tienen durante su turno de trabajo es con robots y no con otros trabajadores ${ }^{15}$. Puede, además, favorecer mayores exigencias mentales y de estrés en el trabajo debido a la aceleración del tiempo, la "infobesidad" o "infoxicación" (sobrecarga informativa) o la aparición de otras patologías en el trabajo como el síndrome del burnout o la adicción al trabajo (workaholism) ${ }^{16}$. También hay que tener en cuenta las

\footnotetext{
${ }^{14}$ FERNÁNDEZ AVILÉs, J. A, "NTC y riesgos psicosociales en el trabajo: estado de situación y propuestas de mejora", op. cit., p. 73.

${ }^{15}$ Disponible en: www.acl.cl/amazon-wearable-monitorear-trabajadores

${ }^{16}$ Vid. el estudio sobre las patologías psicosociales más típicas derivadas del uso de las nuevas tecnologías
} 
características del trabajo en la economía digital ${ }^{17}$ : trabajo basado en el talento; cambiante; conectado; competitivo; entorno digital; inteligente y flexible; y medido en resultados. En efecto, el trabajo del futuro prescindirá de títulos, diplomas o trayectorias acumuladas. Es el talento del individuo lo que cuenta a efectos de empleabilidad y los contenidos de las tareas que realice serán permanentemente cambiantes. En la era del trabajo digital e inteligente los empleados son los dueños de su tiempo. Deciden cuándo y dónde trabajar, lo que se supone que si son capaces de gestionar adecuadamente les concede mayor libertad para conciliar su vida personal y familiar con la laboral ${ }^{18}$. Ante la exigencia de continua adaptabilidad y aprendizaje nos enfrentamos a otro de los problemas generales del tratamiento preventivo de los riesgos psicosociales: ¿Cómo favorecer el cambio tecnológico en la producción sin dejar de respetar el principio ergonómico-personalista (de adaptación del trabajo a la persona)? La gestión del cambio tecnológico y sus efectos sobre los trabajadores debe ser especial objeto de atención en las políticas empresariales de prevención de riesgos laborales ya que constituye un factor adicional de riesgo psicosocial, habría que evitar cambios constantes, lo que parece difícil ante el panorama que se plantea, pero sí se pueden eliminar los innecesarios. ${ }^{19}$.

Los problemas de salud laboral que puede llevar aparejado el uso de las TICs son evitables y tratables, para ello hay que abordar las medidas preventivas que han de ponerse en marcha en las organizaciones ${ }^{20}$. En relación al tiempo de trabajo, la NTP 1123 recomienda, sin carácter exhaustivo, las siguientes:

en FERNÁNDEZ AVILÉS, J. A, "NTC y riesgos psicosociales en el trabajo: estado de situación y propuestas de mejora", op. cit., p. 84 y 85 y SALANOVA et al., "La adicción al trabajo", NTP 759, INSHT, 2007.

${ }^{17}$ Cedrola Sprmolla, G., "El trabajo en la era digital: reflexiones sobre el impacto de la digitalización en el trabajo, la regulación laboral y las relaciones labrales", Revista Internacional y Comparada de Relaciones Laborales y Derecho del Empleo, vol. 5, nº 1, 2017, p. 1-30.

18 Cedrola Spremolla, G, “Competencias, Organización del Trabajo y Formación Profesional en el Trabajo del Futuro: Algunas reflexiones para posibilitar cambios imprescindibles”, Revista Internacional y Comparada de Relaciones Laborales y Derecho del Empleo, vol. 7, nº 1, 2019, p. 32-33.

${ }^{19}$ FERNÁNDEZ AVILÉS, J. A., "NTC y riesgos psicosociales en el trabajo: estado de situación y propuestas de mejora", op. cit., p. 78

${ }^{20}$ Deben ser considerados como riesgos laborales. La Directiva 89/654/CEE establece en el artículo 5.1 que el empresario deberá garantizar la seguridad y la salud de los trabajadores "en todos los aspectos relacionados con el trabajo". Por su parte, el artículo 4.2 de la LPRL define el riesgo laboral como la posibilidad de que un trabajador sufra un determinado daño derivado del trabajo. El informe Mettling visibiliza la problemática que da origen al reconocimiento del derecho a la desconexión en la legislación francesa: trabajadores continuamente conectados y los efectos que ello provoca en su salud, relacionando la conectividad permanente con el derecho a la salud, desde un enfoque preventivo. AAVV, "Transformación digital y vida laboral: las propuestas del informe Mettling", Actualidad Internacional Sociolaboral, $\mathrm{n}^{\circ}$ 194, (última consulta 5/3/2019), Disponible en: www.mitramiss.gob.es. También ese enfoque aparece en el Informe del Comité Económico y Social: CES, Papel y perspectivas de los interlocutores sociales y otras organizaciones de la sociedad civil en el contexto de las nuevas formas de trabajo, 2017, (última consulta: 25/5/2019), Disponible en: httpps: //eur-lex.europa.eu 
- Diseñar una guía de buenas prácticas para el uso adecuado de las TICs, hacer campañas de sensibilización en la empresa para trabajadores, mandos intermedios, superiores jerárquicos etc., evitar las comunicaciones e interacciones entre compañeros de trabajo y superiores jerárquicos (mediante el uso de llamadas telefónicas, correo electrónico etc.) fuera de la jornada laboral.

- Establecer políticas de desconexión digital para garantizar los tiempos de descanso entre jornadas laborales (por ejemplo, instalando sistemas restrictivos de desconexión automática que inhabilite la recepción y el envío de correos electrónicos fuera de la jornada laboral para evitar una "jornada laboral sin fin").

- Planificar el trabajo a realizar, la cantidad de trabajo previsible y el inesperado, así como el tiempo de dedicación previsible y el plazo de finalización.

- Establecer prioridades de actuación en caso de recibir tareas no esperadas para evitar prolongar el tiempo de trabajo y la multitarea.

- Diseñar un procedimiento para llevar a cabo interacciones con trabajadores remotos para evitar la comunicación una vez acabada la jornada laboral.

- Programar adecuadamente el tiempo de trabajo y/o la franja horaria destinada a gestionar el uso del correo electrónico, evitando la multitarea y las interrupciones constantes en el desarrollo de la actividad.

- Evitar la excesiva multiplicidad de localizaciones. Tener en cuenta la distancia entre ellas. Programar el tiempo de trabajo contando con el tiempo invertido en los desplazamientos.

Es en este contexto de hiperconexión digital en el que surge el derecho a la desconexión digital, recientemente regulado en nuestra legislación.

\section{El nuevo derecho a la desconexión digital}

En la actualidad, aproximadamente un tercio de la fuerza de trabajo en el mundo $(36,1 \%)$ realiza un gran número de horas, entendiéndose por horas excesivas de trabajo las que superan de forma habitual las 48 horas por semana. A menudo, los trabajadores necesitan hacer horas de más para complementar unos salarios bajos, y quienes están en esta situación están sobrerrepresentados en los países en desarrollo. Si bien los hombres son más propensos a trabajar un número excesivo de horas, ello no significa que las mujeres no pasen muchas horas de más realizando tareas en el hogar y cuidando de la familia. En uno u otro caso, las horas excesivas de trabajo pueden provocar problemas de salud laboral $^{21}$. En este sentido, el informe de 2019 de la Comisión Mundial sobre el Futuro del Trabajo de la OIT señala que: "el establecimiento de límites a jornadas de trabajo

\footnotetext{
${ }^{21}$ OIT, Seguridad y Salud en el centro del futuro del trabajo. Aprovechar 100 años de experiencia, 2019, (última consulta 10/6/2019), Disponible en: https://www.ilo.org/safework/events/safeday/WCMS_686762/lang--es/index.htm, p. 49
} 
excesivamente largas reducirá los accidentes del trabajo y los riesgos psicosociales asociados a dichas jornadas" 22 . También en España, con un máximo de 40 semanales de trabajo efectivo de promedio en cómputo anual (artículo 34 del TRET ${ }^{23}$ ), es un hecho la prolongación no reconocida de la jornada de trabajo. Según datos del INE correspondientes al primer trimestre del año 2019, los asalariados realizan cada semana un total de 2636 horas extraordinarias no remuneradas por las empresas ${ }^{24}$. Un dato aún más preocupante si se tienen en cuenta las horas de trabajo no remuneradas en el hogar. En este caso, según la Encuesta Nacional de Condiciones de Trabajo 2015 las mujeres dedican 63,6 horas semanales frente a las 56,7 de los hombres ${ }^{25}$.

A nivel europeo ${ }^{26}$ y a fin de garantizar un equilibrio entre seguridad y flexibilidad laboral se firma por los interlocutores sociales europeos en el año 2002 el Acuerdo Marco Europeo sobre Teletrabajo, que fue revisado en $2009^{27}$. En 2017 se previó la regulación del derecho a la desconexión digital en el Código de Trabajo Francés ${ }^{28}$, en el que se remite a la negociación anual sobre igualdad profesional entre mujeres y hombres y calidad de vida en el trabajo y se asigna al empresario un importante papel ya que tiene que concretar a falta de acuerdo con el comité de empresa y previa audiencia del mismo o, en su defecto, de los delegados de personal, la política de actuación al respecto ${ }^{29}$. En Italia, por su parte, se incluyó en la Ley 81 de 2017 la denominada reforma Jobs Act con la que se pretende la "identificación de los períodos de descanso del trabajador, así como las medidas técnicas y organizativas necesarias para garantizar la desconexión del trabajador del equipo de trabajo tecnológico", sin establecer obligaciones concretas a cumplir por el empresario ${ }^{30}$. En otros países como Alemania se ha barajado la posibilidad

22 OIT, Trabajar para un futuro más prometedor, 2019, (última consulta: 11/6/2019), Disponible en: https://www.ilo.org/safework/events/safeday/WCMS_686762/lang--es/index.htm,

${ }^{23}$ Real Decreto Legislativo 2/2015, de 23 de octubre, por el que se aprueba el texto refundido de la Ley del Estatuto de los Trabajadores.

${ }^{24}$ Última consulta: 13/6/2019, Disponible en: www.ine.es

${ }^{25}$ Incluyendo en ambos casos además de las horas no remuneradas los desplazamientos. (última consulta 14/6/2019), Disponible en: www.ine.es

${ }^{26}$ En el ámbito internacional destaca Filipinas en el que se prevé el derecho a la desconexión digital en el Proyecto de Ley 4721 estableciendo el derecho de los empleados de desconectarse de las comunicaciones electrónicas relacionadas con el trabajo después de las horas de trabajo (artículos 48-A y 49B).

${ }^{27}$ La aplicación del mismo es voluntaria para los Estados miembros por lo que toma importancia su regulación en la negociación colectiva.

${ }^{28}$ El artículo 55. 12 ${ }^{\mathrm{a}}$ L 2016-1088 introduce el apartado 7 en el artículo L 2242-8 del Código de Trabajo francés.

${ }^{29}$ La regulación francesa del derecho a la desconexión digital fue por primera vez objeto de estudio y atención en ALEMAN PÁIEZ, F., "El derecho de desconexión digital: una aproximación conceptual, crítica y contextualizadora al hilo de la "Loi Travail no 2016-1088"”, Trabajo y Derecho, no 30, 2017.

${ }^{30}$ Legge 22 maggio 2017, $\mathrm{n}^{\mathrm{o}} .81$. Misure per la tutela del lavoro autónomo non imprenditoriale e misure volte a favorire l'articulazione flessibile nei tempi e nei loughi del lavoro subordinato. El artículo 19 en concreto, y en general el capítulo II regula el denominado "lavoro agile" (trabajo ágil o "smart work") 
de regular el derecho a la desconexión ${ }^{31}$ y son, precisamente, algunas de sus empresas las que han sido pioneras en la implementación de políticas de desconexión (Volkswagen, Allianz, Telekom, Bayer o Henkel).

En España, el artículo 88 de la LO 3/2018 de 5 de diciembre sobre Protección de Datos Personales y Garantía de Derechos Digitales (LOPDP) regula por primera vez el derecho a la desconexión digital en el ámbito laboral de los trabajadores y empleados públicos a fin de garantizar, fuera del tiempo de trabajo legal o convencionalmente establecido, el respeto de su tiempo de descanso, permisos y vacaciones, así como de su intimidad personal y familiar ${ }^{32}$. La Disposición Final $13^{\mathrm{a}}$ de la LOPDP introduce un artículo 20bis en el ET en el que se reconoce "el derecho de los trabajadores " a la intimidad en el uso de los dispositivos digitales puestos a su disposición por el empleador, a la desconexión digital y a la intimidad frente al uso de los dispositivos de video vigilancia y geolocalización en los términos establecidos en la legislación vigente en materia de protección de datos personales y garantía de los derechos digitales".

La doctrina había advertido de que debía de evitarse el peligro de que las medidas de prevención de riesgos psicosociales para los trabajadores con uso intensivo de TICs acabasen integrando el área de responsabilidad social corporativa a partir de la unilateralidad empresarial, reclamando para ello que se reforzase el papel de los representantes de los trabajadores ${ }^{33}$. En la nueva regulación, y siguiendo la estela del

como una nueva manera de ejercer de forma flexible el trabajo subordinado con el objetivo de aumentar la productividad de los empleados y facilitar la conciliación de la vida familiar y profesional.

${ }^{31}$ En el año 2013, la ministra alemana de trabajo Ursula von der Leyen alegó que la desconexión digital protegería la salud mental de las trabajadoras. En el año 2014, la nueva ministra de trabajo, Andrea Nahles valoró una nueva legislación antiestrés en la que se regulara el derecho a la desconexión digital para proteger la salud mental de los trabajadores. Ya en el año 2018 se ha pensado en la posibilidad de normativizar la desconexión digital.

${ }^{32}$ La regulación del derecho a la desconexión digital tiene consideración de Ley ordinaria según la Disposición final primera de la LO 3/2018, de 5 de diciembre, de Protección de datos personales y garantía de los derechos digitales. TODOLí señala que podría haber una contradicción en la norma ya que la DF 13 -que añade un nuevo artículo 20.bis en el ET reconociendo el derecho a la desconexión digital- tiene naturaleza de Ley orgánica, esto es, desarrolla un derecho fundamental. Así se pregunta cómo es posible que el derecho a la desconexión digital pueda ser a la vez ley orgánica y ley ordinaria. La respuesta, añade, parece que vendría por entender que el derecho a la desconexión digital proviene de un Derecho Fundamental, pero que, su desarrollo, conforme a la doctrina del TC, al no ser limitativa de derecho, puede realizarse por ley ordinaria. El reconocimiento de la existencia de vinculación directa entre el derecho a la desconexión y el derecho fundamental determina, entre otras, las consecuencias de un despido de un trabajador por ejercer su derecho a la desconexión. Todolí, A. "El derecho a la desconexión digital aprobada por la LOPDGDD y la prevención de riesgos laborales”, Blog: Argumentos en Derecho Laboral, (última consulta: 1/6/2019), https://adriantodoli.com/blog.

${ }^{33}$ FERNÁNDEZ AVILÉS, J. A, "NTC y riesgos psicosociales en el trabajo: estado de situación y propuestas de mejora", op. cit., p..98. 
ordenamiento jurídico francés, la negociación colectiva se convierte en el ámbito natural de la regulación de la desconexión digital y habrá de precisar su alcance ${ }^{34}$. Dos son las interpretaciones del alcance de la llamada a la negociación colectiva. Podría entenderse que el derecho a la desconexión digital sólo podrá ejercitarse si está regulado -y conforme se regule- en un convenio colectivo o un protocolo interno, quedando vacío de contenido en caso de que no exista dicha regulación. Se trataría por tanto de un derecho cuya realización se condiciona a su desarrollo en convenio colectivo o acuerdo informal de empresa $\mathrm{y}$, previsiblemente, también en un pacto estatutario o en cualquier otra manifestación de la autonomía colectiva ${ }^{35}$. Sin embargo, la norma no impone un deber de negociar ni se ha optado por incluir esta cuestión en el contenido necesario del convenio ${ }^{36}$. Así pues, la segunda interpretación entendería que el derecho a la desconexión digital existe de manera absoluta, pero que la negociación colectiva puede matizar ese ejercicio conforme a la naturaleza y objeto de la relación laboral y sector concreto. En este sentido, el primer epígrafe fijaría un derecho a la desconexión; el segundo indicaría que ese derecho es modulable por la negociación colectiva; y el tercer epígrafe establecería obligaciones para el empresario en materia de prevención de riesgos laborales ${ }^{37}$. Ya han empezado a vislumbrarse en los convenios colectivos en España las primeras referencias al derecho a la desconexión, aunque no siempre refiriendo dicha terminología. Así, por ejemplo, el Convenio colectivo de Vueling Airliness SA (2017) ${ }^{38}$. no menciona explícitamente el derecho a la desconexión digital, pero excluye de la obligación de revisión del correo electrónico los periodos vacacionales, enfermedad, permisos y días libres. El Convenio colectivo del grupo AXA $(2017)^{39}$ fue el primero en establecer el derecho a la desconexión digital y reconoce el derecho de los trabajadores a no responder a los correos o mensajes profesionales fuera de su horario laboral. El Acuerdo para la

\footnotetext{
${ }^{34}$ Garcia-Perrote Escartín, I y Mercader Uguina, J.R., "El permanente debate sobre la jornada laboral: una cuestión clásica (reducción del tiempo de trabajo) y otra reciente (el derecho a la desconexión del trabajo), Revista de Información Laboral, n 10, 2016, Aranzadi Insignis, BIB 2016/80442.

${ }^{35}$ Resulta destacable el Acuerdo Interprofesional de Cataluña para los años 2018-2020 que aborda nuevas problemáticas del mercado laboral, entre ellas, el derecho a la desconexión digital a la finalización de la jornada. En este sentido, IgARTUA considera que va a resultar más eficaz el impulso desde los acuerdos interprofesionales o las recomendaciones y la formación de los negociadores en este terreno. IGARTUA MIRÓ, M Ta "El derecho a la desconexión en la Ley Orgánica 3/2018, de 5 de diciembre de protección de datos personales y garantía de los derechos digitales", Revista de Trabajo y Seguridad Social, CEF, 2019, $\mathrm{n}^{\circ} 432$, p.77.

${ }^{36}$ TASCÓN LÓPEZ, R., "El derecho de desconexión de los trabajadores (potencialidades en el ordenamiento español), Trabajo y Derecho: Nueva Revista de Actualidad y Relaciones Laborales, $\mathrm{n}^{\circ} 41, \mathrm{p} .8$

${ }^{37}$ TODOLí, A. "El derecho a la desconexión digital aprobada por la LOPDGDD y la prevención de riesgos laborales", op., cit.

${ }^{38}$ Resolución de 22 de marzo de 2017, de la Dirección General de Empleo, por la que se registra y publica el Convenio colectivo de Vueling Airlines, S.A (BOE nº 80 de 4 de abril).

${ }^{39}$ Resolución de 21 de septiembre de 2017, de la Dirección General de Empleo, por la que se registra y publica el Convenio colectivo del Grupo Axa (BOE nº. 244, de 10 de octubre).
} 
distribución de la jornada en Ikea Ibérica $(2018)^{40}$ reza lo siguiente: "Los trabajadores tienen derecho a no responder a cualquier tipo de comunicación por cualquier canal (...) fuera de su horario de trabajo, salvo causa de fuerza mayor. Se realizará un seguimiento de tal medida". También el Acuerdo Banco Santander, Banco Popular y Banco Pastor $(2018)^{41}$ al objeto de pactar medidas de adaptación de las relaciones laborales al nuevo entorno digital y asegurar el tiempo de vacaciones y descanso, así como la vida personal y familiar. Por su parte, el Convenio colectivo del grupo Maritima Dávila SA (2018) ${ }^{42}$ conecta la desconexión con el teletrabajo y el VII Convenio colectivo de Repsol SA (2018) con la conciliación de la vida familiar y personal. Más recientemente, el Acuerdo suscrito entre el grupo telefónica y CCOO y UGT $(2019)^{43}$ vinculado al ámbito del bienestar digital y hábitos saludables en el buen uso de las tecnologías digitales. En todo caso, desde el ámbito sindical se ha reivindicado una regulación legislativa de mínimos del derecho a la desconexión digital a fin de garantizar la protección de todos los trabajadores, sin perjuicio de las mejoras que puedan ser introducidas de la mano, fundamentalmente, de la negociación colectiva. De esta forma, se denuncia que a pesar de que la inclusión en el RD Legislativo 2/2015, de 23 de octubre, por el que se aprueba el texto refundido de la Ley del Estatuto de los Trabajadores del derecho a la desconexión digital es una buena iniciativa, su falta de regulación puede llevarle a la situación en la que se encuentran los riesgos psicosociales en los que su control en las empresas españolas ha sido muy desigual, en función del grado de regulación convencional y lo avanzado de ésta en la materia ${ }^{44}$.

Por otro lado, no incluye la norma una definición del derecho a la desconexión digital, aunque la finalidad del mismo, como no podía ser de otra forma, es garantizar, fuera del tiempo de trabajo legal o convencionalmente establecido, el respeto de su tiempo de descanso, permisos y vacaciones, así como de su intimidad personal y laboral. El tiempo de trabajo es un elemento estratégico en el ámbito laboral, para el empresario porque es clave la existencia de un mínimo de horas que han de ser trabajadas para garantizar una adecuada productividad en la empresa y para el trabajador, por el contrario, para que pueda descansar por razones de salud, disfrute del tiempo libre o conciliación. Sin una intervención reguladora externa, la tendencia natural del empresario, como se vio durante los siglos XIX y XX (con jornadas de 10 y 12 horas diaria) es la de apropiarse de la fuerza de trabajo el mayor tiempo posible, con la idea de obtener mayor productividad. Sin

\footnotetext{
40 (última consulta (3/6/2019), Disponible en: https://www.ccoo-servicios.es/archivos/comercio/Acuerdohorario-ikea.pdf

${ }^{41}$ (última consulta 22/5/2019), Disponible en: https://www.ccoo-servicios.es/.../santander/

${ }^{42}$ BOE de 11 de octubre de 2018.

${ }^{43}$ BOE de 17 de julio de 2018.

${ }^{44}$ UGT, Incidencia de las nuevas tecnologías de la información y de la comunicación en la seguridad y salud de los trabajadores, Secretaría de Salud Laboral y Medio Ambiente UGT.CEC, 2019, p. 36 y 37.
} 
embargo, se ha demostrado que esta mayor productividad no depende necesariamente del número de horas, sino que hay otros factores también determinantes ${ }^{45}$. Profusa es la legislación internacional y europea sobre jornada de trabajo y derecho al descanso ${ }^{46}$. En España, el derecho al descanso se regula en el apartado $2^{\circ}$ del artículo 40 de la $\mathrm{CE}^{47}$ y refieren al tiempo de trabajo y tiempo de descanso los artículos 34 a 38 del TRET. También lo hace el Real Decreto 1561/1995 sobre jornadas especiales de trabajo, así como otras normas de rango reglamentario para sectores concretos. En todo caso, deben ser los convenios colectivos los que prevean las concreciones o limitaciones de la jornada de trabajo. También se va sentando jurisprudencia que relaciona la conexión permanente y los excesos de control por parte del empresario como la SAN de 17 de julio $1997^{48}$ que ya estableció que no se puede obligar a los trabajadores a una conexión permanente con la empresa a través de sus teléfonos móviles o la STS de 21 de septiembre de $2015^{49}$ que recuerda que los trabajadores no están obligados a facilitar a la empresa sus teléfonos o correos electrónicos. Si bien es cierto que en este caso estamos ante una exigencia contraria a la protección de datos y no puede considerarse precursor del derecho a la desconexión digital. Respecto a los periodos de disponibilidad o guardias localizadas la STSJUE de 21 de febrero de 2018 (Ville de Nivelles vs Rudy Matzak. Asunto C-518) interpreta el artículo 15: "no pueden los Estados miembros adoptar definiciones del tiempo de trabajo más restrictivas, pero sí más favorables para los trabajadores". Y el artículo 2: "No es tiempo de trabajo cuando a pesar de hallarse en guardia localizada no se requiere la presencia en el lugar de trabajo". Por su parte, la STSUE de 5 de octubre de 2004 (Asunto C-397/2001): "No se puede superar la jornada máxima añadiendo periodos de disponibilidad, aunque no sean siempre tiempos de trabajo efectivos incluyendo periodos en los que no existe actividad laboral'. Y en España sobre la misma cuestión la STS de 27 de enero de $2005^{50}$ (y jurisprudencia posterior): "las guardias localizadas con llamada de trabajo son consideradas tiempo de trabajo, no las que no tienen llamada".

Ante el deber de abstención por parte del empresario, se ha dudado de si estamos ante un derecho o ante un derecho-deber del trabajador, reconociéndose que, aunque no parece posible penalizar al trabajador por incumplimiento de sus deberes relativos a la

\footnotetext{
${ }^{45}$ Mella Mendez, L., "El impacto de los convenios de la OIT sobre la retribución, tiempo de trabajo, seguridad y salud y terminación del contrato en el derecho español: puntos críticos", Revista de Trabajo y Seguridad Social, CEF, 434, p..68.

${ }^{46}$ Vid al respecto y sobre el derecho a la desconexión digital vid: SERRANO ARGÜESO, M., "Always on. Propuestas para la efectividad del derecho a la desconexión digital en el marco de la economía 4.0", Revista Internacional y Comparada de Relaciones Laborales y derecho del Empleo, vol.7, $\mathrm{n}^{\circ}$ 2, abril-junio 2019.

${ }^{47}$ Constitución Española, BOE n ${ }^{\circ} 311$, de 29 de diciembre de 1978.

${ }^{48}$ Sentencia $n^{\circ} .94 / 1997$ de 17 julio AS\1997\3370

${ }^{49}$ STS, $4^{\text {a }}$, de 21 de septiembre de 2015 (recurso 259/2014)

${ }^{50}$ STS, $4^{\text {a }}$, de 27 de enero de 2005, $n^{\circ} .5539 / 2003$, Ref. Cendoj 2005/375
} 
desconexión resultará difícil satisfacer el derecho si no se cuenta con la colaboración del trabajador $^{51}$. La obligación empresarial, de inexcusable cumplimiento, tendrá que ser implementada en las empresas, independientemente de su actividad o tamaño y su omisión podrá encontrar encaje sancionador al albur de la Ley de Infracciones y Sanciones del Orden Social ${ }^{52}$.

No es suficiente con que la empresa permita a los trabajadores disfrutar de los descansos, sino que debe adoptar un rol activo de vigilancia, supervisión, seguimiento y control de que esos descansos no son interrumpidos ${ }^{53}$. En este sentido establece la norma que será el empleador, previa audiencia a los representantes de los trabajadores, quién deba elaborar una política interna dirigida a trabajadores, incluidos los que ocupen puestos directivos, en la que definirán las modalidades de ejercicio del derecho a la desconexión y las acciones de formación y de sensibilización del personal sobre un uso razonable de las herramientas tecnológicas que evite el riesgo de fatiga informática ${ }^{54}$ Dicha previsión en la negociación colectiva pretende atender a la realidad productiva y a las distintas necesidades organizativas de las empresas ${ }^{55}$. Se deja pues en manos del empresario la puesta en marcha de la política, eso sí, de conformidad con lo establecido en el convenio colectivo o acuerdo de empresa y previa audiencia a los representantes ${ }^{56}$. Dada la incidencia en la salud psicosocial sería idóneo que dicha audiencia se tratase a través del Comité de Seguridad y Salud si lo hay o que se consulte a los delegados de prevención. Lo que no establece la ley son las consecuencias de una negativa de los representantes de

\footnotetext{
${ }^{51}$ IgARTUA Miró, Ma . T., "El derecho a la desconexión en la Ley Orgánica 3/2018, de 5 de diciembre de protección de datos personales y garantía de los derechos digitales", op. cit., p. 70.

${ }^{52}$ ZAMORA, S., "Desconexión digital ¿novedad o anécdota?, Diario La Ley, no 9363, Sección Tribuna, 21 de febrero de 2019. IGARTÚA menciona los siguientes: el artículo 7.5 de la LISOS (relativo a la transgresión del tiempo de trabajo); artículo 12.1 de la misma norma referente a la evaluación de riesgos o la realización de las actividades de prevención que hicieran necesario los resultados de las evaluaciones; artículo 8.11 (actos del empresario contrarios al respecto de la intimidad y consideración debida a la dignidad de los trabajadores). IGARTUA MiRÓ, Ma. T., "El derecho a la desconexión en la Ley Orgánica 3/2018, de 5 de diciembre de protección de datos personales y garantía de los derechos digitales", op.cit., p. 70.

${ }^{53}$ TODOLí, A. "El derecho a la desconexión digital aprobada por la LOPDGDD y la prevención de riesgos laborales", op., cit.

${ }^{54}$ BAYLOS GRAU señala que "no basta con un código de conducta interno para la puesta en práctica de esta política, sino que este texto deberá ser necesariamente acordado con los representantes de los trabajadores en coherencia con el mandato legislativo relativo a la regulación del derecho a la desconexión, que está estrechamente ligado con el supuesto de la llamada "fatiga informática"'. BAYLOS GRAU, A., "Una nota sobre el papel de la negociación colectiva en la configuración de los derechos derivadas de la Ley de Protección de Datos Personales y garantía de derechos digitales en España", (última consulta: 6/5/2019), Disponible en: ipeatra@ yahoogrupos.com.br

${ }^{55}$ Rojo Torrecilla, E., "Los derechos digitales laborales en la Ley Orgánica 3/2018, de 5 de diciembre, de Protección de Datos Personales y garantía de los derechos digitales. Notas al título X", Blog: El blog de Eduardo Rojo, (última consulta 30/1/2019), Disponible en: http://www.eduardorojotorrecilla.es.

${ }^{56}$ Frente a la regulación francesa en la española hay libertad de forma.
} 
los trabajadores a aceptar la política empresarial sobre las modalidades de ejercicio del derecho a la desconexión. En todo caso, no es un derecho de veto sino de audiencia.

Por otro lado, establece la norma que se preservará el derecho a la desconexión digital en los supuestos de realización total o parcial del trabajo a distancia, así como en el domicilio del empleado vinculado al uso con fines laborales de herramientas tecnológicas. Reflexiona IGARTUA que habría que hacer, al menos, una tipología del derecho a la desconexión acorde con la necesidad de regular las modalidades de ejercicio divididas entre trabajadores presenciales, a distancia o parcialmente a distancia; trabajadores con horario regular, flexible o con capacidad de autogestión del tiempo de trabajo ${ }^{57}$. En el caso de que el trabajo sea presencial, el problema es el de los límites de la conexión permanente. Cuando el trabajo es a distancia las mayores dificultades están relacionadas con el control de la jornada de trabajo. La inexistencia de un protocolo para la efectiva desconexión digital puede tener importantes consecuencias, como se ha dicho, en la salud de los trabajadores, pudiendo ser posible la sanción en ese ámbito en el caso de demostrarse la conexión entre la falta de medidas por parte de la empresa y el riesgo psicosocial. ${ }^{58}$. En todo caso, pese a la posibilidad de incluir los riesgos psicosociales en las genéricas previsiones contenidas en la LPRL"59 "quizá no estaría de más una actualización legal en este terreno, si bien, en puridad, en relación a la desconexión lo indispensable resulte ser su posterior concreción vía convencional, incluyendo la ubicación sistemática más adecuada"60.

En todo caso, parece que la desconexión podría no ser absoluta ya que al igual que en la legislación francesa se refiere a pactar las condiciones de "uso razonable" de los dispositivos digitales en el ámbito laboral ${ }^{61}$ por lo que las acciones de formación y

${ }^{57}$ La política interna ha de adoptarse en todo caso y podría estar integrada en la propia política de prevención de riesgos laborales obligatoria de la empresa. IGARTUA MIRó, Ma . T., "El derecho a la desconexión en la Ley Orgánica 3/2018, de 5 de diciembre de protección de datos personales y garantía de los derechos digitales", op. cit., p. 69 y 84.

${ }^{58}$ MiÑARRO añade que, en este caso, la Ley debiera haber previsto su inserción en la Ley de Prevención de riesgos laborales, puesto que al tratarse de un nuevo riesgo laboral debe activarse el sistema preventivo evaluación y planificación. MiÑARRO YANINI, M., "La carta de derechos digitales de los trabajadores ya es ley: menos claros que oscuros en la nueva regulación”, Trabajo y Seguridad Social, CEF, $\mathrm{n}^{\circ} 430$, enero 2019, p. 13.

59 14; $15.1 \mathrm{~d} ; 16$ o 19 , todos ellos de las LPRL.

${ }^{60}$ IgARTUA Miró, Ma . T., “El derecho a la desconexión en la Ley Orgánica 3/2018, de 5 de diciembre de protección de datos personales y garantía de los derechos digitales”, op.cit. p. 74. El ETUC había propuesto que temas como el derecho a conectarse, el derecho a desconectarse y la cuestión de protección de datos de los trabajadores deberían "abordarse conjuntamente a nivel europeo para evitar enfoques diferentes y distintas formas de reglamentación. CES, Dialogo Social: programa de trabajo 2018-2020, 13 de junio de 2017

${ }^{61}$ Charro BAEnA, P., "Cambios tecnológicos y tiempo de trabajo", III Encuentro Internacional sobre Transformaciones del Derecho del Trabajo Ibérico. Derechos fundamentales y tecnologías innovadoras, 
sensibilización son especialmente importantes para garantizar el derecho al descanso. En este mismo sentido, parece que la legislación no apuesta por políticas más agresivas para su efectividad como, por ejemplo, podría ser registrar el encendido y apagado de los ordenadores, periodos de siesta digital bloqueando la posibilidad de recibir correos electrónicos, el acceso remoto al servidor de la empresa o la prohibición de utilizar el correo o el teléfono durante una franja horaria determinada una vez acabada la jornada laboral.

El derecho a la desconexión digital garantiza, en resumen, el viejo derecho al descanso que se materializa en conseguir diferenciar de forma adecuada la vida laboral, familiar y personal. Pero, se plantea la doctrina, ¿para ese viaje de regreso al clasicismo jurídico necesitamos estas alforjas esnobistas o a la moda de la era digital? ${ }^{62}$

\section{¿Cómo garantizar el derecho al descanso de los trabajadores y una conciliación corresponsable?}

El uso prolongado o "mal uso" de las TICs alarga la jornada laboral y provoca interferencias $y / o$ invasiones en el tiempo de ocio y descanso incidiendo significativamente en la conciliación de la vida familiar, personal y laboral. La NTP 1122 identifica en relación a este hecho los siguientes riesgos psicosociales: inadecuada gestión de la flexibilidad temporal que caracteriza la realización del trabajo usando TICs; manejo inadecuado de los dispositivos portables, intranet, correo electrónico etc., en cualquier momento; ausencia de una cultura preventiva o guía de buenas prácticas relativas al uso adecuado de las TICs; existencia de expectativas de disponibilidad y respuesta inmediata por parte de los superiores jerárquicos y/o sensación de "deber de contestar" fuera de la jornada laboral por parte de los trabajadores; no existencia de políticas empresariales específicas sobre promoción de trabajadores remotos que puede hacer que aumenten las prácticas laborales inadecuadas como el leaveism al incrementarse la competitividad con objeto de promocionar o usar las TICs fuera de la jornada laboral para llevar el trabajo "al día". Para la efectividad del derecho a la conciliación la empresa debe ser un agente activo en la implementación de la desconexión vigilando, controlando y haciendo seguimiento de que los descansos no son interrumpidos y diseñando una política empresarial clara sobre su uso adecuado en la jornada laboral que incluya entre otros: protocolos, instrucciones, circulares o guías de buenas prácticas en el manejo de las TICs para evitar su uso inadecuado una vez terminada la jornada; no utilizando indiscriminadamente las distintas cuentas de correo electrónico para uso laboral

(última consulta 4/6/2019), Disponible en: https://www.cije.up.pt, p.. 24.

${ }^{62}$ VALLECILlo GómEZ, R., "El derecho a la desconexión: ¿Novedad digital o esnobismo del viejo derecho al descanso?", Trabajo y Seguridad Social. CEF, n 408, 2017, p. 172. Vid. también MolinA NAVARRETE, C., "Desconexión digital. Garantía del derecho al descanso", Temas Laborales, n 138, 2017, p. 249-283. 
(diferenciar la cuenta de correo personal de la laboral); campañas de sensibilización y programas formativos de la empresa para sensibilizar sobre la incidencia en el equilibrio vida-trabajo; o medidas más extremas en casos límites como limitar la posibilidad de usar el correo electrónico de la empresa a determinadas horas (siestas digitales), establecer días sin correo electrónico, garantizar la desconexión digital instalando sistemas de desconexión automática que inhabilite la recepción y envío de correos electrónicos fuera de la jornada laboral etc. ${ }^{63}$. El registro obligatorio de la jornada de trabajo, recientemente incorporado por el Real Decreto-ley 8/2019, de 8 de marzo, de medidas urgentes de protección social y de lucha contra la precariedad laboral en la jornada de ${ }^{64}$ es también una forma de garantizar la no realización de horas extraordinarias (no remuneradas) y, por extensión, la conciliación de la vida laboral con la personal y familiar, a pesar de la dificultad de su implementación en algunos sectores ${ }^{65}$.

Pero no es suficiente garantizar la conciliación entre la vida personal, familiar y laboral ya que si no tendemos a una conciliación corresponsable seguiremos feminizando el cuidado y forzando a las mujeres a realizar dobles jornadas de trabajo. En efecto, las políticas legislativas que hasta ahora se han implementado en España en materia de conciliación de la vida familiar, personal y laboral no han conseguido subvertir ni revolucionar el rol tradicional de la mujer como cuidadora, al contrario, se han fomentado las medidas de ausencia de la persona al puesto de trabajo, en la mayor parte de los casos, de las mujeres madres y cuidadoras en vez de apostar firmemente por medidas de presencia que promuevan la adaptación de la jornada de trabajo y que dejen que sean las

\footnotetext{
${ }^{63}$ MANZano SANTAMaría, N, "Las Tecnologías de la Información y Comunicación (TIC) (II): factores de riesgo psicosocial asociados a las nuevas formas de organización del trabajo", op .cit.

${ }^{64}$ Se modifica el apartado 7 del artículo 34 del ET, que queda redactado de la siguiente manera. " $E l$ Gobierno, a propuesta de la persona titular del Ministerio de Trabajo, Migraciones y Seguridad Social y previa consulta a las organizaciones sindicales y empresariales más representativas, podrá establecer ampliaciones o limitaciones en la ordenación y duración de la jornada de trabajo y de los descansos, así como especialidades en las obligaciones de registro de jornada, para aquellos sectores, trabajo y categorías profesionales que por sus peculiaridades así lo requieran". Se añade un nuevo apartado 9 al artículo 34: "La empresa garantizará el registro diario de la jornada, que deberá incluir el horario concreto de inicio y finalización de la jornada de trabajo de cada persona trabajadora, sin perjuicio de la flexibilidad horaria que se establece en este artículo. Mediante negociación colectiva o acuerdo de empresa o, en su defecto, decisión del empresario previa consulta con los representantes legales de los trabajadores en la empresa, se organizará y documentará este registro de jornada. La empresa conservará los registros a los que se refiere este precepto durante cuatro años y permanecerán a disposición de las personas trabajadoras, de sus representantes legales y de la Inspección de Trabajo y Seguridad Social".

${ }^{65}$ La nueva regulación otorga al empresario una posición de garante de la existencia de un registro diario de la jornada. A la negociación colectiva le corresponde fijar cómo se ha de organizar y documentar ese registro de la jornada y en su defecto, y previa consulta con la representación de los trabajadores al empresario. El incumplimiento de la obligación de registro se incluye como infracción grave en artículo 7.5 de la LISOS siendo de relativa poca entidad la cuantía del incumplimiento pues la infracción es por empresa o centro no individual (entre 626 y 6125 euros).
} 
personas trabajadoras las que puedan coordinar sus condiciones laborales con su vida privada. En este sentido, las políticas europeas de work life balance evidencian la necesidad de desarrollar iniciativas legislativas que aborden desde una perspectiva multidisciplinar el tiempo como eje articulador de la vida laboral y cotidiana ${ }^{66}$. La propuesta de Directiva del Parlamento Europeo y del Consejo relativa a la conciliación de la vida familiar y la vida profesional de los progenitores y cuidadores, por la que se deroga la Directiva 2010/18/UE del Consejo establece el derecho de adaptación de la jornada como un derecho de conciliación real ${ }^{67}$. Sin embargo, como hemos dicho, el derecho a la conciliación en España se ha construido sobre una carencia de corresponsabilidad, sin permisos bien diseñados y sin financiación suficiente ni servicios públicos desarrollados de atención a la infancia y la dependencia que permitan a las personas trabajadoras atender a las necesidades de cuidados familiares sin menoscabo de su participación y desenvolvimiento en el mercado de trabajo. ${ }^{68}$. Ante esta situación, muy recientemente, empiezan a aparecer iniciativas legislativas para una conciliación corresponsable. En el año 2018 se aprueba la proposición de Ley de tiempo de trabajo corresponsable ${ }^{69}$ basada en la necesidad de garantizar un reparto equitativo de tiempos de trabajo y de cuidado, así como un efectivo derecho a la adaptación del trabajo a la persona, a la desconexión del trabajo $\mathrm{y}$, en definitiva, a facilitar la conciliación $\mathrm{y}$ corresponsabilidad entre la vida personal, familiar y laboral, así como la igualdad real y efectiva entre mujeres y hombres teniendo en cuenta, como explica la misma, que el tiempo de trabajo ha sido abordado hasta ahora en nuestro país como un instrumento para el incremento de la flexibilidad empresarial y no desde una perspectiva respetuosa con el cuidado y la corresponsabilidad. Esta propuesta de norma sobre tiempo de trabajo o sobre presencia en el trabajo parte de una obligación de registro de la jornada de trabajo y establece que para su efectividad será preciso acompañar a las medidas propuestas de políticas públicas sobre racionalización de horarios. El Real Decreto Ley 6/2019, de 1 de marzo, de medidas urgentes para la garantía de la igualdad de trabajo y oportunidades entre mujeres y hombres en el empleo y la ocupación ${ }^{70}$ modifica nuestra legislación con un espíritu corresponsable. Es una norma a la que se la ha tildado de precipitada e insuficiente pero no de innecesaria o banal porque refuerza la idea de que la cuestión de la igualdad efectiva entre hombres y mujeres en el trabajo requiere de medidas

\footnotetext{
${ }^{66}$ Chacartegui Jávega, C, "Tiempo de trabajo, racionalidad horaria y género: un análisis en el contexto europeo", La Ley, 2006, n 19, p. 1201.

${ }^{67}$ Propuesta de Directiva del Parlamento Europeo y del Consejo relativa a la conciliación de la vida familiar y la vida profesional de los progenitores y los ciudadanos, y por la que se deroga la Directiva 2010/18/UE del Consejo (COM (2017) 253 final).

${ }^{68} \mathrm{CES}$, Informe sobre participación laboral de las mujeres en España, 2016, p..120.

${ }^{69}$ 122/000299 Proposición de Ley de tiempo de trabajo corresponsable, Boletín Oficial de las Cortes Generales, Congreso de los Diputados, XII Legislatura, Serie B, Proposiciones de Ley, 16 de noviembre de 2018.

${ }^{70} \mathrm{BOE} \mathrm{n}{ }^{\circ} 57$ de 7 de marzo de 2019.
} 
específicas, a pesar de la insuficiencia de sus contenidos y de la necesidad de una reforma global y efectivamente transversal susceptible de avanzar en el ámbito de la igualdad entre hombres ${ }^{71}$. Parece que por fin estamos en el camino hacia la corresponsabilidad, diferente de la conciliación y que requiere un mayor grado de protección al cuidado. En este camino se necesita garantizar la neutralidad de los derechos de conciliación estableciendo la obligación para los hombres de asumirlos. La corresponsabilidad replantea los roles de género en la sociedad; requiere construir una sociedad de roles igualitarios en todos los ámbitos y trasciende el ámbito público para incidir en el privado implicando a varios sujetos: las personas que forman parte de la familia mediante la implicación masculina para el reparto del trabajo doméstico y de cuidado; la empresa, quien debe aceptar esta nueva redistribución de responsabilidades y disponer de una gestión adecuada del tiempo de trabajo productivo que permita la continuidad de las personas en sus puestos de trabajo; y la propia sociedad que contribuye mediante modificaciones legislativas e institucionales ${ }^{72}$. Es por ello que una de los cambios más importantes y esperanza en el avance hacia la corresponsabilidad ha sido la reforma del artículo 34.8 del TRET fuertemente criticado en la legislación anterior por su supeditación a la negociación colectiva, y calificada ahora tanto de interesante como de inquietante. En la redacción actual se pretende garantizar la existencia del derecho a solicitar la adaptación de la jornada reconociendo directamente a las personas trabajadoras que "tienen derecho a solicitar las adaptaciones (...) para hacer efectivo su derecho a la conciliación de la vida familiar y laboral", y desvinculando su existencia de una eventual regulación convencional que se limita a regular "los términos de su ejercicio" que deberán acomodarse a "criterios y sistemas que garanticen la ausencia de discriminación, tanto directa como indirecta, entre personas trabajadoras de uno y otro sexo"73. El RDL amplia también el objeto del derecho y, además de la duración y distribución de la jornada abarca "la ordenación del tiempo de trabajo y la forma de la prestación, incluida la prestación del trabajo a distancia" Ello, no obstante, elimina la referencia a la vida personal que sí tenía la anterior regulación. Además, la redacción es confusa en cuanto a los sujetos que pueden solicitar el derecho de adaptación ${ }^{74}$. Según BALLESTER

\footnotetext{
${ }^{71}$ BALLESTER PASTOR, M. A, "El RDL 6/2019 para la garantía de igualdad de oportunidades entre mujeres y hombres en el empleo y la ocupación: Dios y el diablo en la tierra del sol", Femeris, vol. 4, no 2, p.15.

72 Ballester Pastor, M. A., "La era de la corresponsabilidad: los nuevos retos de la política antidiscriminatoria", Lan Harremanak, n 25, 2012, p. 56.

${ }^{73}$ PASTOR MARTINEZ, A., "Las medidas laborales del Real Decreto-Ley 6/2019, de 1 de marzo, de medidas urgentes para garantía de la igualdad de trabajo y oportunidades entre mujeres y hombres en el empleo y la ocupación: un paso hacia la efectividad de la igualdad de trabajo y de oportunidades desde la corresponsabilidad", IUSLabor, n 1, 2019, p. 204.

${ }^{74}$ La redacción del artículo confunde en cuanto a si sólo se puede solicitar el derecho por cuidado de hijos hasta 12 años o si se puede solicitar por cuidado, pero en el caso de que sea por hijos será hasta que tengan 12 años. Uno y otro caso más limitativos que la redacción anterior por lo que necesitaría una mejora de su redacción.
} 
PASTOR es el tercer párrafo el que "suscita inquietud cuando establece un procedimiento meticuloso de concesión que parece más desconfiado que garantista y que refuerza la malévola, pero generalizada percepción de que los beneficios por cuidado de dependientes serán previsiblemente objeto de abuso en perjuicio de la empresa y del resto de trabajadores por parte de trabajadores (generalmente trabajadoras) sin demasiados escrúpulos". Y continua "mucho más relevante hubiera sido una referencia a la necesidad de que la organización del tiempo de la empresa y de las personas trabajadoras se realizara con carácter global, teniendo en cuenta no sólo las necesidades de conciliación sino también las formativas (en coherencia con el texto estatutario) y tomando en consideración que la organización del tiempo de trabajo de todas las personas trabajadoras es una materia de prevención de riesgos laborales ${ }^{75}$ que obliga a la consideración de los riesgos psicosociales, las necesidades de los trabajadores sensibles y la situación especial de las madres en situación de embarazo, maternidad y lactancia"76.

Algunos de los aspectos más positivos de las TICs bien utilizadas son la flexibilidad horaria y la posibilidad de conciliar mejor la vida laboral con la personal y familiar, su mal uso, sin embargo, como hemos visto, vulnera la legislación al realizarse más horas de las legalmente permitidas, perjudica la salud psicosocial de las personas trabajadoras e impide la conciliación de la vida personal, familiar y laboral. Es por ello por lo que son imprescindibles políticas concretas que den un paso más que la simple reivindicación del viejo derecho al descanso, promuevan el bienestar en el trabajo e incluso entren en ámbito privado. Las políticas de conciliación de nueva generación deben basarse y partir de la corresponsabilidad y de la distribución igualitaria de los roles de la vida, no solo en el ámbito laboral sino también en los ámbitos personal y familiar (descanso, ocio, formación deporte, familia...) para los que la persona necesita disponer de tiempo, por ello no es redundante y sí necesaria la garantía del derecho a la desconexión digital y las medidas políticas y legislativas que se adopten en este sentido, como el registro de la jornada de trabajo. No debe descargarse en el trabajador la decisión de conectarse o no fuera de su tiempo de trabajo, sino que debe incidirse en la obligación empresarial de respetar los tiempos preestablecidos de descanso y, por lo tanto, de desconexión ${ }^{77}$. La empresa ha de asumir que el bienestar en el trabajo es una inversión económica que ahorra costes en términos de reducción del absentismo, mejora de la productividad, reduce la accidentalidad, beneficia la calidad del servicio prestado o trabajo realizado, evita

\footnotetext{
${ }^{75}$ Esta naturaleza tiene la Directiva 2003/88 de 4 de noviembre de ordenación del tiempo de trabajo.

${ }^{76}$ BALLESTER PASTOR, M. A, "El RDL 6/2019 para la garantía de igualdad de oportunidades entre mujeres y hombres en el empleo y la ocupación: Dios y el diablo en la tierra del sol”, op. cit., p.15 y 16.

${ }^{77}$ UGT, Incidencia de las nuevas tecnologías de la información y de la comunicación en la seguridad y salud de los trabajadores, op. cit., p. 36.
} 
perjuicios en la imagen corporativa y ahorra sanciones e indemnizaciones por daños en la salud de trabajadores derivados de falta preventiva ${ }^{78}$.

\section{Bibliografía}

AA.VV., "Transformación digital y vida laboral: las propuestas del informe Mettling", Actualidad Internacional Sociolaboral, no 194, (última consulta 5/3/2019), Disponible en: www.mitramiss.gob.es.

Aleman PÁIEZ, F., "El derecho de desconexión digital: una aproximación conceptual, crítica y contextualizadora al hilo de la "Loi Travail no 2016-1088"', Trabajo y Derecho, $n^{\circ} 30,2017$.

BAYLOS GRAU, A., "Una nota sobre el papel de la negociación colectiva en la configuración de los derechos derivadas de la Ley de Protección de Datos Personales y garantía de derechos digitales en España", (última consulta 6/5/2019), Disponible en: ipeatra@yahoogrupos.com.br.

BALlester PAstor, M. A., "La era de la corresponsabilidad: los nuevos retos de la política antidiscriminatoria", Lan Harremanak, n² 25, 2012, p. 53-77.

BALLESTER PASTOR, M. A, "El RDL 6/2019 para la garantía de igualdad de oportunidades entre mujeres y hombres en el empleo y la ocupación: Dios y el diablo en la tierra del sol", Femeris, Vol. 4, no 2, pp. 14-38.

Cedrola Spremolla, G., "El trabajo en la era digital: reflexiones sobre el impacto de la digitalización en el trabajo, la regulación laboral y las relaciones laborales", Revista Internacional y Comparada de Relaciones Laborales y Derecho del Empleo, vol. 5, $\mathrm{n}^{\mathrm{o}} 1$, 2017, p.1-30.

Cedrola Spremolla, G, "Competencias, Organización del Trabajo y Formación Profesional en el Trabajo del Futuro: Algunas reflexiones para posibilitar cambios imprescindibles", Revista Internacional y Comparada de Relaciones Laborales y Derecho del Empleo, vol. 7, nº 1, 2019, p. 1-51.

Centro Nacional de Condiciones de Trabajo, "El futuro de la prevención", NTP 1000, INSHT, 2014.

\footnotetext{
${ }^{78}$ FERNÁNDEZ AVILÉS, J. A, "NTC y riesgos psicosociales en el trabajo: estado de situación y propuestas de mejora", op. cit., p. 99.
} 
CES, Informe sobre participación laboral de las mujeres en España, 2016.

CES, Dialogo Social: programa de trabajo 2018-2020, 13 de junio de 2017.

CES, Papel y perspectivas de los interlocutores sociales y otras organizaciones de la sociedad civil en el contexto de las nuevas formas de trabajo, 2017, (última consulta 25/5/2019), Disponible en: https: //eur-lex.europa.eu.

ChaCARTEGUi JÁVEGA, C, “Tiempo de trabajo, racionalidad horaria y género: un análisis en el contexto europeo", La Ley, 2006, n 19, p. 1201-1223.

Charro Baena, P., "Cambios tecnológicos y tiempo de trabajo", III Encuentro Internacional sobre Transformaciones del Derecho del Trabajo Ibérico. Derechos fundamentales y tecnologías innovadoras, (última consulta: 4/6/2019), Disponible en: https://www.cije.up.pt.

EDENRED-IPSOS, Bienestar y motivación de los empleados, junio 2015, (última consulta 4/6/2019), Disponible en www.edenred.es/descarga-barometro-edenred-ipsos.

FERNÁNDEZ AVILÉS, J. A., "NTC y riesgos psicosociales en el trabajo: estado de situación y propuestas de mejora", DSL, 2-2017, p. 69-101.

Garcia-Perrote Escartín, I y Mercader Uguina, J.R., "El permanente debate sobre la jornada laboral: una cuestión clásica (reducción del tiempo de trabajo) y otra reciente (el derecho a la desconexión del trabajo), Revista de Información Laboral, n 10, 2016, Aranzadi Insignis, BIB 2016/80442.

HERNÁNDEZ, J.A et al, "Las tecnologías de la Información y Comunicación y la Brecha Digital: Una nueva forma de exclusión social”, Boletín Científico de la Escuela Superior de Atotonilco de Tula, $\mathrm{n}^{\circ}$ 11, 2019, (última consulta 15/5/2019), Disponible en: https//repository.uaeh.edu.mx/revistas/index.php/Atotonilco/issue/ archive.

IgARTUA Miró, $M^{a}$ T, "El derecho a la desconexión en la Ley Orgánica 3/2018, de 5 de diciembre de protección de datos personales y garantía de los derechos digitales", Revista de Trabajo y Seguridad Social, CEF, 2019, n 432, p. 61-87.

Instituto Nacional de Seguridad, Salud y Bienestar en el Trabajo (INSSBT), Algunas orientaciones para evaluar los factores de riesgo psicosocial, Torrelaguna (Madrid), 2015. 
MANZANO SANTAMARíA, N, "Las tecnologías de la Información y la Comunicación (TIC)(I): nuevas formas de organización del trabajo", NTP 1122, INSBT, 2018.

MiÑARRO Y ANINI, M., "La carta de derechos digitales de los trabajadores ya es ley: menos claros que oscuros en la nueva regulación", Trabajo y Seguridad Social, CEF, n 430, 2019 , p. 5-14.

Mella Mendez, L., "El impacto de los convenios de la OIT sobre la retribución, tiempo de trabajo, seguridad y salud y terminación del contrato en el derecho español: puntos críticos", Revista de Trabajo y Seguridad Social, CEF, n 434, p. 57-96.

Molina NAVARrete, C., "Desconexión digital, Garantía del derecho al descanso", Temas Laborales, $\mathrm{n}^{\circ} 138,2017$, p. 249-283.

OIT, Seguridad y Salud en el centro del futuro del trabajo. Aprovechar 100 años de experiencia, 2019, (última consulta 10/6/2019), Disponible en: https://www.ilo.org/safework/events/safeday/WCMS_686762/lang--es/index.htm.

OIT, Trabajar para un futuro más prometedor, 2019, (última consulta 11/6/2019), Disponible en: https://www.ilo.org/safework/events/safeday/WCMS_686762/lang-es/index.htm.

Pastor MartineZ, A., "Las medidas laborales del Real Decreto-Ley 6/2019, de 1 de marzo, de medidas urgentes para garantía de la igualdad de trabajo y oportunidades entre mujeres y hombres en el empleo y la ocupación: un paso hacia la efectividad de la igualdad de trabajo y de oportunidades desde la corresponsabilidad", IUSLabor $\mathrm{n}^{\circ} 1$, 2019, p. 187-209.

RoJo TorReciLla, E., "Los derechos digitales laborales en la Ley Orgánica 3/2018, de 5 de diciembre, de Protección de Datos Personales y garantía de los derechos digitales. Notas al título X", Blog: el blog de Eduardo Rojo, (última consulta 30/01/2019), Disponible en: http://www.eduardorojotorrecilla.es.

SALANOVA et al., "La adicción al trabajo", NTP 759, INSHT, 2007.

Serrano Argüeso, M., "Always on. Propuestas para la efectividad del derecho a la desconexión digital en el marco de la economía 4.0", Revista Internacional y Comparada de Relaciones Laborales y derecho del Empleo, vol. 7, nº 2, abril-junio 2019.

TASCÓN LÓPEZ, R., "El derecho de desconexión de los trabajadores (potencialidades en 
el ordenamiento español), Trabajo y Derecho: Nueva Revista de Actualidad y Relaciones Laborales, $\mathrm{n}^{\mathrm{o}}$ 41, p. 1-31.

TODOLÍ, A. "El derecho a la desconexión digital aprobada por la LOPDGDD y la prevención de riesgos laborales", Blog: Argumentos en Derecho Laboral, (última consulta: 1/6/2019), https://adriantodoli.com/blog.

UGT, Incidencia de las nuevas tecnologías de la información y de la comunicación en la seguridad y salud de los trabajadores, Secretaría de Salud Laboral y Medio Ambiente UGT.CEC, 2019.

VALLECILLO GÓMEZ, R., "El derecho a la desconexión: ¿Novedad digital o esnobismo del viejo derecho al descanso?", Trabajo y Seguridad Social, CEF, n 408, 2017, p. 167-178.

Zamora, S., "Desconexión digital ¿novedad o anécdota?, Diario La Ley, no 9363, Sección Tribuna, 21 de febrero de 2019. 